\title{
Herpesviruses and heparan sulfate: an intimate relationship in aid of viral entry
}

\author{
Deepak Shukla and Patricia G. Spear \\ Department of Microbiology-Immunology, Northwestern University Medical School, Chicago, Illinois, USA \\ Address correspondence to: Patricia G. Spear, Department of Microbiology-Immunology, Mail Code S213, \\ Northwestern University Medical School, 320 East Superior Street, Chicago, Illinois 60611, USA. \\ Phone: (312) 503-8230; Fax: (312) 503-1339; E-mail: p-spear@northwestern.edu. \\ J. Clin. Invest. 108:503-510 (2001). DOI:10.1172/JCI200113799.
}

Glycosaminoglycan chains on cell surface proteoglycans provide initial docking sites for the binding to eukaryotic cells of various viruses and other microorganisms. The ubiquitously expressed glycosaminoglycan heparan sulfate is particularly important in this regard, at least for viruses. Often, binding of a viral protein to heparan sulfate is only the first step in a cascade of interactions between virus and cell that is required for viral entry into the cell and the initiation of infection. The steps that occur after binding of virus to heparan sulfate may require the interaction of other viral proteins with other cell surface molecules.

Several of the human herpesviruses, the focus of this Perspective, can make their initial contact with cells by binding to cell surface heparan sulfate. One member of this group, herpes simplex virus type 1 (HSV-1), can also bind to specific sites in heparan sulfate to trigger the fusion between viral envelope and cell membrane that is required for viral entry.

\section{The family of herpesviruses}

The herpesvirus family is large and contains more than 100 members that infect organisms from oysters to humans. Herpesvirus particles consist of a large linear double-stranded DNA genome, ranging in size from about 100,000 to 250,000 base pairs; an icosahedral capsid, which packages the genome and has 162 morphological units; and a layer of proteins called the tegument, which surrounds the capsid and is in turn enclosed within a lipid-containing envelope. To replicate, herpesviruses take over both the nucleus and cytoplasm of the infected cell, which is usually killed in the process. These viruses also have the capacity to establish nonlytic, latent infections in cells that, under certain conditions, do not support viral replication. In latently infected cells, the viral genome is stably associated with the cell nucleus and expresses few if any viral proteins. There is little or no pathology associated with latent infections, but activation of the latent viral genomes may occur due to changes in the state of the cell, resulting in new rounds of virus replication and new episodes of disease.
Eight herpesviruses, differing markedly in their biology, are known to infect humans. These include herpes simplex types 1 and 2 (HSV-1, HSV-2), varicella-zoster virus (VZV), cytomegalovirus (CMV), Epstein-Barr virus (EBV), human herpesvirus 6 (HHV-6), HHV-7, and HHV-8, or Kaposi sarcoma-associated herpesvirus. Based on genomic analysis and other biologic characteristics, the herpesviruses are classified into three subfamilies. The alphaherpesviruses, including HSV-1, HSV-2, and VZV, are neurotropic cytolytic viruses that can establish asymptomatic latent infections in neurons of the peripheral nervous system. The betaherpesviruses include cytomegalovirus (CMV) - so named because infected cells become massively enlarged or "cytomegalic." This subfamily also includes HHV-6 and HHV-7. Members of the gammaherpesvirus subfamily are lymphotropic and include EBV and HHV-8. Both HHV-8 and EBV are considered important cofactors in malignancies.

\section{Clinical manifestations of herpesvirus diseases in humans}

Infections with each of the eight human herpesviruses, if one includes latent infections, are highly prevalent in all human populations. The clinical manifestations of infection must be divided into two categories: those evident after primary infection (primary disease) and those resulting from activation of latent virus (reactivation disease). The importance of cell-mediated immunity in controlling herpesvirus replication and in limiting reactivation of latent virus in vivo is apparent from the dramatic increase in severity and frequency of disease in immunodeficient persons. The transmission of herpesviruses usually requires intimate contact between persons, such as kissing or sexual intercourse. Often, the transmitting person is an asymptomatic shedder of infectious virus. Among the human herpesviruses, HSV has the broadest host range. Although HSV is normally isolated only from humans, many animal species can be experimentally infected, and many types and species of cultured cells will support HSV replication. In contrast, the other human herpesviruses have a much more 
limited host range, are fastidious about the cell types in which they will replicate, and are often much more difficult to propagate in cell culture. For all the herpesviruses, the ability to establish latent infections and to reactivate from latency are key to their ability to persist for the lifetime of the infected host, to maintain a high prevalence in most human populations, and to cause significant disease long after the initial infection and primary disease, if any.

The most common form of disease caused by HSV in humans is manifested as mucocutaneous lesions, which occur usually in or near the mouth (cold sores or fever blisters), on the cornea (keratitis), or on genital tissues. Because the virus that causes the primary lesions establishes latent infections in sensory or autonomic peripheral neurons and can be reactivated by appropriate stimuli, periodic recurrences of herpetic lesions are common and present one of the troublesome aspects of infections with HSV. Less frequently, HSV can also cause lifethreatening disease affecting vital organs, including encephalitis in apparently normal adults and disseminated disease in infants and immunocompromised individuals. Virus reactivation and replication may occur periodically in asymptomatic persons. Although HSV-1 and HSV-2 can infect the same body sites and cause indistinguishable lesions, the viruses are mostly associated with different sets of diseases. The viruses isolated from cases of adult encephalitis, keratitis, and facial lesions are usually HSV-1, whereas those isolated from cases of neonatal disease, adult meningitis, and genital lesions are usually HSV-2.

Like HSV, VZV can replicate in the epidermis and establish latent infections in neurons. In contrast to HSV, VZV is transmitted in epidemic fashion, via aerosols and the respiratory route, and causes a systemic rather than local primary disease. Varicella or chicken pox has the hallmarks of an acute viral disease affecting the entire lymphoreticular system with a generalized vesicular eruption of the skin and mucosal membranes. VZV establishes latent infections in sensory nerves. Reactivation of virus is infrequent and occurs usually in older individuals, resulting in zoster or shingles, an eruption of skin lesions that is limited to a single dermatome and often causes severe pain.

CMV infections are typically asymptomatic. The most common clinical manifestation is a self-limited mononucleosis, although disease can be life-threatening in immunodeficient individuals. CMV can cause hepatitis, chorioretinitis, pneumonitis, colitis, and congenital cytomegalic inclusion disease and can be a contributing factor to failure of organ transplantation. The cell types that harbor latent virus are probably of the monocytic lineage. The major means of CMV transmission are by the congenital, oral, and sexual routes and by blood transfusion or tissue transplantation. The other two human members of the betaherpesvirus family, HHV-6 and HHV-7, are T lymphotropic and are both capable of causing a childhood illness called sixth disease or roseola infantum. Most children become infected with these viruses, but very few show clinical manifestations of disease.
Primary infections of children with EBV are usually asymptomatic, but, in young adults, the consequence can be infectious mononucleosis. The virus establishes latent infections in B lymphocytes and can induce proliferation of B cells through expression of a limited number of viral genes. A competent immune system keeps these latently infected B cells in check, but abrogation of cell-mediated immunity can result in lymphoproliferative disease. The other human member of the gammaherpesvirus subfamily, HHV-8, is the cause of Kaposi sarcoma, a vascular tumor of mixed cell composition, and body cavity-based lymphoma found in AIDS patients.

It is thus evident that the human herpesviruses differ markedly in their ability to infect various cell types and to establish productive or latent infections in these cell types.

\section{Pathways of herpesvirus entry into cells}

Enveloped viruses enter cells by inducing fusion between the viral envelope and a cell membrane. This membrane fusion can be triggered in at least two ways, resulting in different pathways of entry. For example, the binding of a virus to a cell may induce endocytosis of the virus, followed by acidification of the endosome, which can trigger fusion between the viral envelope and endosome membrane. Alternatively, the binding of a virus to a cell may result in multiple receptor-ligand interactions at the cell surface that can trigger fusion between the viral envelope and the plasma membrane. Most herpesviruses apparently enter cells via the latter pathway. They can fuse directly with the cell plasma membrane. Fusion might also occur after endocytosis of the virus particle in an early endosome, but is not dependent on the acidification of endosomes.

The herpesvirus envelope is a lipid bilayer derived from a host cell membrane in which most cell proteins have been displaced by viral membrane proteins. As many as a dozen or more integral membrane proteins and glycoproteins encoded by the virus become incorporated into the viral envelope. For most herpesviruses, only four or five of the envelope proteins may participate in the process of viral entry. Rigorous proof that a viral envelope protein has a role in viral entry comes from characterizing the specific infectivity of a viral mutant that produces virions devoid of the protein in question. A viral mutant that cannot produce a protein required for viral entry cannot, of course, be propagated. Such mutants can be propagated, however, in a cell line transformed to express the missing protein (a complementing cell line) so that the protein is supplied in trans for viral assembly. Passage of the virus once through a noncomplementing cell line results in production of virus particles missing the protein in question, and these particles can be assessed for their ability to enter cells.

By constructing and characterizing many such HSV-1 mutants, it has been possible to identify five viral glycoproteins $(\mathrm{gB}, \mathrm{gC}, \mathrm{gD}, \mathrm{gH}$, and $\mathrm{gL})$ that contribute to viral entry (1). Four of them, $g B, g D, g H$, and $\mathrm{gL}$, are essential for viral entry. In the absence of any one of these glycoproteins, the mutant virions can bind 
to cells but fail to enter them. Absence of $\mathrm{gC}$ results in reduced binding of virus to cells, although the virus that binds can enter cells and initiate infection (2). Absence of both $\mathrm{gB}$ and $\mathrm{gC}$ severely reduces the binding of HSV-1 to cells (3). These findings, and others discussed below, indicate that either $\mathrm{gC}$ or $\mathrm{gB}$ can mediate the binding of virus to cells, whereas $\mathrm{gB}, \mathrm{gD}, \mathrm{gH}$, and $\mathrm{gL}$ are all required for the membrane fusion that leads to viral entry (Figure 1).

Because HSV-1 and HSV-2 are so closely related, it has been assumed, although not rigorously shown, that the related HSV-2 glycoproteins (with the same names) have similar roles in viral entry. For the other human herpesviruses, the generation of appropriate viral mutants has been much more difficult to achieve, and the viral glycoproteins required for entry have, in general, not been identified. It has been possible, however, to express soluble forms of individual viral glycoproteins that are candidates for having a role in entry, to determine whether they bind to cell surfaces and compete with virus for binding or entry.

All the human herpesviruses, regardless of subfamily, express glycoproteins that are evolutionarily related to HSV gB, gH, and gL. Considerable attention has focused on these glycoproteins because of the possibility that evolutionary relatedness in gene and protein sequence implies at least some functional relatedness. Indeed, for most herpesviruses, $\mathrm{gB}$ is probably required for viral entry and probably binds to cell surface molecules, including heparan sulfate in several cases. For most herpesviruses, heterodimers of $\mathrm{gH}-\mathrm{gL}$ are probably also required for viral entry, in some cases as heterotrimers with yet an additional viral protein in the complex. Most alphaherpesviruses, including HSV-1, HSV-2, and related animal viruses, but excluding VZV, express a member of the $\mathrm{gD}$ family, and this member is required for viral entry. Most alphaherpesviruses also express a member of the $\mathrm{gC}$ family, and this member usually mediates the binding of virus to cells through interactions with heparan sulfate, as will be discussed more fully below. Both $\mathrm{gC}$ and $\mathrm{gD}$ appear to be restricted to the alphaherpesvirus lineage.

\section{Heparan sulfate as the initial receptor}

for the binding of herpesviruses to cells

Many different cell surface molecules can serve as receptors for the attachment and entry of viruses. The particular receptor or receptors a virus can use will determine the cell types it can infect and therefore its portal of entry, spread, and pathogenesis. A single virus may have evolved to interact with several cell surface molecules in a cascade of receptor-ligand interactions required for entry. Also, a single virus may have evolved to use different receptors for entry into different cell types. Both possibilities are a reality for at least some of the herpesviruses. To conclude that a particular cell surface molecule is the initial binding receptor for a virus, most or all of the following criteria should be met.

First, absence or removal of the putative receptor from the cell surface should reduce the binding of virus and render the cell at least partially resistant to viral infec- tion. Second, expression of the putative receptor in a cell that does not normally bind virus should allow virus binding and, perhaps, entry. Third, a soluble form of the cell surface receptor should bind to virus and inhibit the binding of virus to receptor-bearing cells. Fourth, a specific viral protein or component of the virus surface should bind to the cell surface receptor in situ and in purified form. Finally, in the case of enveloped viruses, the deletion of the viral ligand from the envelope should render the virus incapable of binding to its receptor or to cells bearing its receptor. Clearly, these rules cannot apply in all cases, since some cells will express multiple receptors for a given virus and, conversely, some viruses will express multiple ligands that are each sufficient to mediate viral binding to cellular receptors.

For HSV-1, cell surface heparan sulfate and probably some forms of dermatan sulfate satisfy the above criteria. Cells treated with heparinases or altered by mutations that prevent heparan sulfate biosynthesis have reduced capacity to bind virus and are at least partially resistant to virus infection $(4,5)$. In fact, HSV-1 or HSV-2 can be used to select for cells mutated in enzymes required for heparan sulfate biosynthesis (or dermatan sulfate biosynthesis, if heparan sulfate is absent) (6-8) and to screen for cells in which the mutations have been complemented (9), because cells become more resistant or susceptible to HSV cytotoxicity, depending on whether they express heparan sulfate. Soluble heparin and appropriate forms of heparan sulfate can inhibit HSV-1 infection, and virus can be shown to bind to heparin $(4,10)$. Both $\mathrm{gB}$ and $\mathrm{gC}$ extracted from virions and soluble forms of $\mathrm{gB}$ and $\mathrm{gC}$ bind to heparin or heparan sulfate or both $(2,11-13)$. Soluble forms of $\mathrm{gC}$ have been shown to inhibit virus

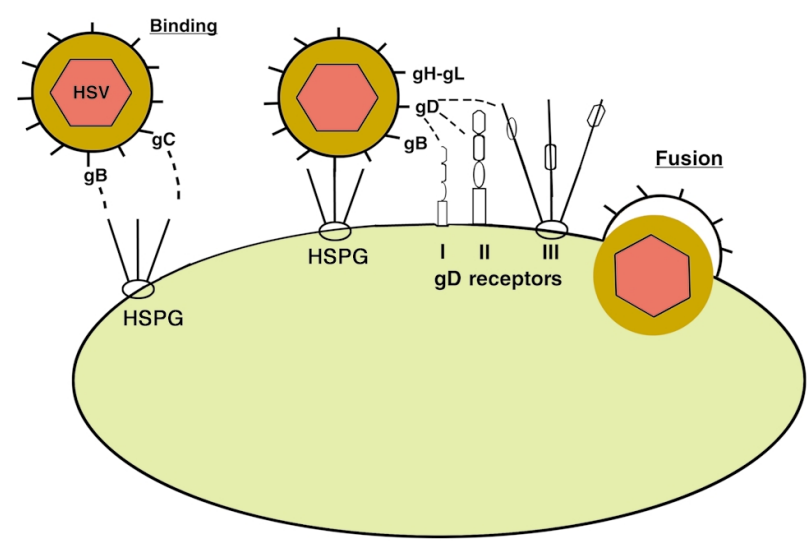

\section{Figure 1}

Entry of HSV-1 into cells. The initial contact of virus with cell is usually the binding of virus to heparan sulfate chains on cell surface proteoglycans (HSPG). Either of the viral glycoproteins gB or gC can mediate this binding. Then viral gD can bind to any one of several entry receptors, including HVEM (I), a member of the TNF-receptor family; nectin-1 or nectin-2 (II), two related members of the immunoglobulin superfamily; or sites generated in heparan sulfate by the action of specific 3-O-sulfotransferases (III). The binding of gD to one of its receptors activates viral membranefusing activity, which requires the action of $\mathrm{gB}$ and $\mathrm{gH}-\mathrm{gL}$ as well as $\mathrm{gD}$ and a gD receptor. Fusion between the viral envelope and cell membrane liberates the viral nucleocapsid and tegument into the cell cytoplasm. 
Table 1

Heparin-binding proteins of the human herpesviruses and roles in viral entry

\begin{tabular}{|c|c|c|c|c|}
\hline Subfamily & Virus & Glycoprotein ${ }^{A}$ & Role in entry & Other comments \\
\hline \multirow[t]{4}{*}{ Alpha- } & HSV-1 (and HSV-2) & $\mathrm{gC}$ & $\begin{array}{l}\text { Binds heparan sulfate to } \\
\text { mediate virus binding to cells }\end{array}$ & Binds C3b \\
\hline & & gB & $\begin{array}{l}\text { Binds heparan sulfate to mediate virus } \\
\text { binding to cells; required for entry }\end{array}$ & $\begin{array}{l}\text { Participates in membrane fusion for entry, } \\
\text { independent of heparan sulfate-binding activi }\end{array}$ \\
\hline & & $\mathrm{gD}$ & $\begin{array}{l}\text { Binds } 3 \text {-O-sulfated heparan } \\
\text { sulfate to mediate entry }\end{array}$ & Also binds protein receptors to mediate entry \\
\hline & VZV & gB (gpll) & $\begin{array}{l}\text { Binds to heparan sulfate; } \\
\text { soluble } g B \text { can inhibit infection }\end{array}$ & \\
\hline \multirow[t]{4}{*}{ Beta- } & CMV & gB & $\begin{array}{l}\text { Binds to heparan sulfate; } \\
\text { soluble gB can inhibit infection }\end{array}$ & $\begin{array}{l}\text { Binds to another receptor to induce } \\
\text { interferon-responsive genes }\end{array}$ \\
\hline & & $\mathrm{gM}(\mathrm{gC}-\mathrm{II})$ & $\begin{array}{l}\text { Binds to heparan sulfate; probably the principal } \\
\text { ligand for heparan sulfate in virus binding }\end{array}$ & $\begin{array}{l}\text { Another viral glycoprotein forms part of the } \\
\text { heparan sulfate-binding complex }\end{array}$ \\
\hline & $\mathrm{HHV7}$ & gB & Binds to heparin and cell surface heparan sulfate & \\
\hline & & gp65 & Binds to heparin and heparan sulfate & \\
\hline
\end{tabular}

${ }^{A}$ Alternative names of the glycoproteins are shown in parentheses.

binding to cells $(11,14)$. Absence of $\mathrm{gC}$ from virions reduces the ability of virus to bind to cells (2) and absence of both $\mathrm{gB}$ and $\mathrm{gC}$ virtually eliminates binding (3). The binding that occurs in the absence of $\mathrm{gC}$ remains dependent on cell surface heparan sulfate. Thus, it has been concluded that the initial interaction of HSV with cells is binding to cell surface heparan sulfate and that $\mathrm{gB}, \mathrm{gC}$, or both can mediate this binding.

Similar but not identical results have been obtained with HSV-2. Binding of HSV-2 and infection is at least partially dependent on cell surface heparan sulfate by the same criteria as applied for HSV-1, and both gB and $\mathrm{gC}$ extracted from virions can bind to heparin-affinity columns (15). The only HSV-2 mutant studied to date is one deleted for $\mathrm{gC} \mathrm{(15).} \mathrm{This} \mathrm{mutant} \mathrm{can} \mathrm{bind} \mathrm{to} \mathrm{cells}$ as well as wild-type virus and exhibited similar specific infectivity, in contrast to the gC-negative HSV-1 mutants. It appears that HSV-1 and HSV-2 differ with respect to the relative importance of their heparin-binding glycoproteins for the initial step of virus binding. Although VZV expresses homologues of gB and gC, it has been difficult to determine whether these glycoproteins play a role in virus binding to cells. Part of the problem is the difficulty of obtaining infectious virus from cultured cells. VZV is one of the most contagious of human herpesviruses, and, clearly, highly infectious virus is excreted from the respiratory tract. When the virus is propagated in cultured cells, however, very little infectious virus is produced, and what is produced may not have the properties characteristic of transmissible virus. Generally, VZV is propagated in vitro by the coculture of intact infected cells with fresh uninfected cells, not by passage of cell-free virus. Despite these technical difficulties, Zhu et al. (16) have shown that heparin can inhibit the binding of cell-free VZV to cells, that the undersulfation of glycosaminoglycans caused by chlorate ions makes cells resistant to VZV infection, and that VZV can bind to heparin-affinity columns. Deletion of $\mathrm{gC}$ from the virus has little effect on viral infection and does not make the infection process less sensitive to heparin inhibition (16). VZV gC may nevertheless have heparan sulfate-binding activity, since deletion of HSV gC (which clearly is a heparan sulfate-binding protein) has variable effects on viral infectivity and also does not make virus binding or infection less sensitive to heparin inhibition. As with HSV gB, VZV gB has been found to bind heparin, and a soluble form of $\mathrm{gB}$ binds efficiently to cells expressing heparan sulfate but poorly to cells devoid of this glycosaminoglycan (17). Moreover, VZV binding and entry are delayed in the presence of the soluble $\mathrm{gB}$. Thus, it seems likely that heparan sulfate has a role as a receptor for VZV binding.

The initial interaction of human CMV with cells is binding to cell surface heparan sulfate. Virus binding and infection are reduced by soluble heparin and heparan sulfate, as well as in cells treated with heparinases or those unable to produce heparan sulfate (18, 19). Two heparin-binding glycoproteins have been identified $(18,20)$. One is the CMV form of $g B$. The other is a complex of proteins originally called gC-II and now recognized to be composed of at least two viral proteins, including $\mathrm{gM}$, a membrane glycoprotein that appears to have the heparin-binding domain (21). All herpesviruses studied to date express a form of gM, but only in the case of human CMV has the protein been reported to have heparin-binding activity. It remains uncertain whether $\mathrm{gB}$ or $\mathrm{gM}$ in the gC-II complex mediates the binding of virus to cells. The construction and characterization of viral mutants devoid of these glycoproteins have not yet been reported.

In the case of HHV-7, heparin can inhibit the binding of virus to cells and can also inhibit viral infection and virus-induced syncytium formation (22). These findings are not sufficient to conclude that the virus binds to cell surface heparan sulfate. Studies with the appropriate heparan sulfate-deficient cells are needed. 
It has been shown, however, that two HHV-7 glycoproteins, $\mathrm{gB}$ and a novel glycoprotein called gp 65 , can bind to heparin and heparan sulfate $(22,23)$. By analogy with other herpesviruses, it seems likely that binding of these glycoproteins to heparan sulfate has a role in the binding of virus to cells. It is unclear whether HHV-6 interacts with cell surface heparan sulfate. Binding of virus to $T$ cells and infection of the cells are only partially inhibited by heparin or heparan sulfate and only at high concentrations (24).

The binding of EBV to B cells can be explained by interactions of gp350/220 with CD21 and gH-gL-gp42 with class II MHC molecules (25). There is no evidence that heparan sulfate has any role in EBV binding or entry. Binding of HHV-8 (the other known human gammaherpesvirus) to cells, however, appears to be dependent largely on interactions of virus with cell surface heparan sulfate (26).

\section{Molecular interactions between herpesvirus glycoproteins and heparan sulfate}

Table 1 lists the known heparin-binding glycoproteins expressed by the human herpesviruses. For HSV-1 gB and $\mathrm{gC}$, both typical type I membrane glycoproteins, there is some information as to structural features of the proteins that are important for binding to heparin or heparan sulfate. Deletion of a short lysine-rich region (KPKKNKKPK) from HSV-1 gB revealed that this sequence is required for binding to heparin and heparan sulfate and for gB-mediated binding of virus to cells, but not for other aspects of $\mathrm{gB}$ function in viral entry (27). Although there is very little variability in overall gB sequence within a serotype (98-99\% identity) or between HSV-1 and HSV-2 (85\% identity), most of the variability is seen in this lysine-rich region. It is tempting to speculate that this variability could dictate recognition of different structural features in heparan sulfate by each form of $\mathrm{gB}$. In fact, there is some evidence to suggest that HSV-1 and HSV-2 recognize different structural features of heparan sulfate when binding to cells, although it is difficult to assess the relative contributions of $\mathrm{gB}$ and $\mathrm{gC}$ to these serotype differences. Certain modified forms of heparin, altered by chemical desulfation, inhibited HSV-2 binding and infection better than HSV-1 binding and infection, regardless of whether HSV-1 expressed gC (28). These results suggest that 2,3-O-sulfation and 6-O-sulfation are critical for binding of heparin to HSV-1 gB but not to the HSV-2 glycoproteins. Consistent with these results, Trybala et al. (13) found that 2-O-sulfation and 6-O-sulfation were more important for the binding to heparin to isolated HSV$1 \mathrm{gB}$ than to isolated HSV-2 gB. In another study, Feyzi and colleagues showed that short fragments of heparin that bound to isolated HSV-1 gC were enriched for these modifications (29). More work needs to be done to identify the precise structural features of heparan sulfate recognized by the $\mathrm{gBs}$ and $\mathrm{gCs}$ encoded by HSV-1 and HSV-2.

The sequences of HSV-1 and HSV-2 gC are highly conserved within each serotype but differ between serotypes, particularly in the $\mathrm{N}$-terminal half where there are many amino acid differences and a large gap in the HSV-2 sequence. Efforts to identify a heparinbinding domain of HSV-1 gC suggest that several regions contribute to this binding. Soluble recombinant forms of HSV-1 gC have been shown to bind to wild-type but not heparan sulfate-negative cells, an interaction that can be markedly but not completely inhibited by heparin (11). Deletion of a 91-amino acid stretch in HSV-1 gC significantly reduces the binding to cells but does not interfere with binding of the altered protein to a heparin-affinity column, suggesting that distinct regions of $\mathrm{gC}$ govern binding to heparin and heparan sulfate. In other studies, Trybala and colleagues (30) noted that mouse red cells can be agglutinated by HSV-1 due to the interaction of $\mathrm{gC}$ with heparan sulfate-like molecules on the red cell. Using this hemagglutination assay, they showed that missense mutations in HSV-1 gC, mapping to two noncontiguous regions within the protein, reduce or abolish this interaction. The mutant viruses had been selected for their resistance to neutralization by one of three different anti-gC mAb's and two of these three Ab's inhibited the hemagglutination. Finally, peptides matching the sequences of the two regions defined by the viral mutations inhibit the binding of virus to cells and infection (31). Taken together, these results indicate that at least three regions of HSV-1 gC may influence binding to heparin or heparan sulfate.

To follow the interactions of CMV gB with cell surface receptors, Boyle and Compton (32) developed a soluble recombinant form of the protein. Cell-binding studies suggested the existence of two types of receptors for $\mathrm{CMV} \mathrm{gB}$, one with an estimated $K_{\mathrm{d}}$ for its interaction with gB of $0.04-0.3 \mu \mathrm{m}$ and the other $5-10 \mu \mathrm{m}$. Interestingly, while both receptor types were evident on human fibroblasts and wild-type Chinese hamster ovary $(\mathrm{CHO})$ cells, heparan sulfate-negative $\mathrm{CHO}$ cells exhibit only the low-affinity class of binding sites. Since soluble gB at relatively low concentrations can inhibit the binding of virus to cells, it appears that this protein blocks binding of virus to heparan sulfate. Binding of either soluble $\mathrm{gB}$ or intact $\mathrm{CMV}$ virions can induce the expression of interferon-responsive genes, but this response does not depend on the presence of cell surface heparan sulfate $(33,34)$. The possibility exists that others of the heparin-binding glycoproteins listed in Table 1 may bind to more than one cell surface molecule, with multiple effects on the cells.

\section{Heparan sulfate as an entry receptor}

Viral entry sometimes requires interactions of viral proteins with coreceptors or entry receptors that are distinct from those that mediate initial virus binding. Such entry receptors are probably required by all the herpesviruses. The search for these receptors can be greatly facilitated by identification of a cell line to which virus binds but fails to penetrate due to lack of entry receptors. For example, taking advantage of the fact that $\mathrm{CHO}$ cells are resistant to infection by HSV-1, we recently introduced pools of human cDNAs and screened for plasmids that could render the cells susceptible to viral entry. It was then possible to identify the cell surface molecules that 

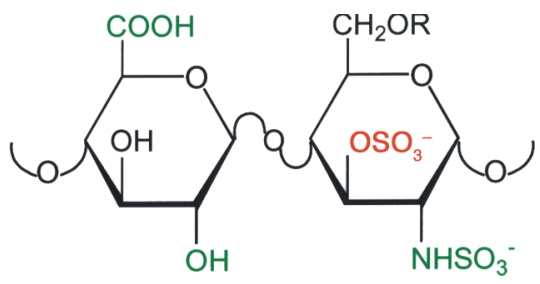

3-OST-1

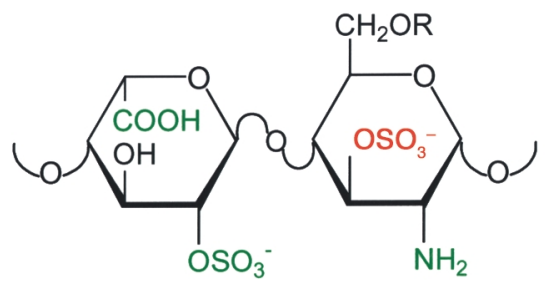

3-OST-3

Figure 2

Structural features of disaccharides in heparan sulfate within the sites modified by 3 -OST- 1 and $3-$ OST- $3_{\text {A }}$ or $3-$ OST- $3_{B}$. Substrate recognition by each enzyme probably involves binding to more than two residues in the polymer, but the groups colored in green may contribute to recognition by each enzyme. The sulfate group added by each enzyme is shown in red. The disaccharide found in 3-OST-1-modified heparan sulfate is part of the pentasaccharide to which antithrombin binds. The disaccharide found in 3-OST-3-modified heparan sulfate is part of the binding site for $\mathrm{gD}$, the size and overall structure of which is not yet known.

served as entry receptors and the viral ligand for these receptors, which proved to be $\mathrm{gD}$. Surprisingly, at least three classes of human gene products could each independently bind to $\mathrm{gD}$ to serve as entry receptors (35). These include a member of the TNF-receptor family named HVEM (HveA) (36), two members of the immunoglobulin family designated nectin-1 (HveC) (37) and nectin-2 (HveB) (38), and a specific modification in heparan sulfate that generates sites for the binding of $\mathrm{gD}$ (39). Any one of these gD receptors is sufficient to mediate viral entry. The interaction of gD with one of its receptors triggers fusion between the viral envelope and cell membrane, which requires the participation of $\mathrm{gB}$ and $\mathrm{gH}-\mathrm{gL}$ as well as $\mathrm{gD}$ and its receptor.

The sites in heparan sulfate that can serve as gD receptors for entry are functional only for HSV-1 and are generated by the action of specific 3-O-sulfotransferases (3-OSTs) (39). The biosynthesis of heparan sulfate requires a chain of reactions, including synthesis of the repeating disaccharide (glucuronic acid and $N$-acetylglucosamine) polymer, $\mathrm{N}$-deacetylation and $\mathrm{N}$-sulfation of selected glucosamine residues, epimerization of selected glucuronic moieties to iduronic acid, 2-O-sulfation of selected iduronic acid residues, and 6-O-sulfation and/or 3-O-sulfation of selected glucosamine residues (40). At least five isoforms of 3-OSTs (3-OST-1, 3-OST-2, 3 -OST- $3_{\mathrm{A}}, 3-\mathrm{OST}-3_{\mathrm{B}}$, and 3 -OST-4) are encoded by the same number of genes in humans and mice (41-43). Whereas 3-OST-1 has been shown to generate binding sites for antithrombin $(41,43), 3-O S T-3_{\mathrm{A}}$ and 3 -OST- $3_{\mathrm{B}}$ generate binding sites for HSV-1 gD $(39,43)$. Accordingly, $\mathrm{CHO}$ cells expressing 3-OST-1 remain resistant to HSV- 1 entry whereas $\mathrm{CHO}$ cells expressing $3-\mathrm{OST}-3_{\mathrm{A}}$ or $3-\mathrm{OST}-3_{\mathrm{B}}$ are susceptible to HSV-1 entry. Moreover, a sol- uble recombinant form of HSV- $1 \mathrm{gD}$ binds directly to 3-OST- $3_{\mathrm{A}}$-modified heparan sulfate with a $K_{\mathrm{d}}$ of about $2 \mu \mathrm{M}$. Interestingly, the $K_{\mathrm{d}} \mathrm{s}$ for binding of soluble $\mathrm{gD}$ to soluble forms of the protein receptors, HVEM or nectin1 , are also in the micromolar range $(44,45)$. While the precise structure of the $\mathrm{gD}$-binding site generated in heparan sulfate by $3-\mathrm{OST}-3_{\mathrm{A}}$ or $3-\mathrm{OST}-\mathrm{3}_{\mathrm{B}}$ is not yet known, part of the structure can be deduced from disaccharide analysis of heparan sulfate modified by these enzymes, which appear to be identical in substrate specificity. Figure 2 shows the structure generated by the 3-OST-3s in comparison with that generated by 3-OST-1. Interestingly, 3-OST-2 and 3-OST-4 have also been found to generate entry receptors for HSV-1 (D. Shukla et al., unpublished results). The substrate specificities for these two enzymes overlap with that of the 3 -OST-3s $(43,46)$. The 3-OST-3s are expressed in a variety of tissues whereas 3-OST-2 and 3-OST-4 are expressed principally in the brain (42). This latter finding is of great interest, considering the association of HSV-1 with sporadic cases of adult encephalitis.

The fact that specific sites in heparan sulfate generated by 3-OSTs can serve as entry receptors for HSV-1 suggests that, for HSV-1 at least, viral binding and entry can be mediated entirely by heparan sulfate, provided the appropriate sites for virus binding and $\mathrm{gD}$ binding are present. It cannot be ruled out, of course, that some ubiquitously expressed but unidentified protein also participates in the entry process when $\mathrm{CHO}$ cells are made to express a 3-OST in the absence of any known protein receptor. Obviously, cells made susceptible to entry via expression of 3-OSTs must express heparan sulfate and also the enzymes, such as 2-OST, required to generate the substrate for the 3-OSTs (39). When cells are susceptible to entry via the expression of protein receptors for $\mathrm{gD}$, however, expression of heparan sulfate is not an absolute requirement for viral binding and entry. Even under these conditions, however, the presence of cell surface heparan sulfate greatly enhances viral binding and entry $(6,7)$. When cell surface proteins that can serve as $\mathrm{gD}$ receptors are overexpressed in the absence of heparan sulfate, virus binding may be mediated, albeit inefficiently, by these receptors or by other unknown receptors. For cells expressing endogenous gD receptors, it seems likely that the numbers of such receptors will be too low to permit much virus binding and that coexpression of heparan sulfate will be a de facto requirement for virus binding and entry.

There is no evidence that any of the other human herpesviruses can use sites in heparan sulfate as anything other than initial binding receptors. Cell surface proteins that can serve as entry receptors have been identified, however, as described above for EBV and summarized elsewhere for CMV (47), HHV-6 (48), and HHV-7 (49).

\section{Implications of herpesvirus binding} to heparan sulfate for pathogenesis

The first step in virus attachment to host cells is governed largely by the probability that a virion and a cell will come close enough to make a contact, which depends not only on the trafficking and concentration 
of free virions and exposure to a cell surface, but also on the presence of physical forces of attraction between the two. The existence of an electrostatic or ionic interaction between herpes virions and cells can be inferred from the fact that the initial binding of virions to cell surfaces is very sensitive to low $\mathrm{pH}$ or high concentration of salts and a variety of polyanions and polycations. Subsequent irreversible binding relies, very likely, more on hydrophobic and other short-range forces whose strength and specificity are determined largely by the conformations of the interacting viral and cellular interfaces. An understanding of which cell types are susceptible to herpesvirus entry will depend on assessing availability of the various binding and entry receptors on exposed cell surfaces. Heparan sulfate, if present on the proteoglycans of exposed cell surfaces, makes an ideal snare for the capture of most herpesviruses and may facilitate subsequent interactions with other coreceptors required for entry. Depending on the nature of interaction and size of heparan sulfate chain, a single chain may bind multiple viral ligands on a virion. For example, heparan sulfate chains on human HEp-2 cells averaged 105 $\mathrm{kDa}(50)$ in apparent molecular mass, corresponding roughly to 420 sugar residues per chain (or $190 \mathrm{~nm}$ in length), which could easily adhere to almost half of the virion circumference (13).

Paradoxically, binding of virus to heparan sulfate could both enable the virus to bind to cells bearing entry receptors and also prevent the virus from reaching such cells. Heparan sulfate is a constituent not only of cell surface proteoglycans, but also of the extracellular matrix and basement membranes in organized tissues. HSV-1 can be found concentrated on the basement membrane in skin punch biopsies infected in organ culture (51). Also, the basal lamina is a physical barrier to HSV-1 infection of mature muscle fibers (52). In addition, certain body fluids contain both heparin and heparin-binding proteins, either of which can inhibit the binding of HSV to cells (4). Thus, the spread of HSV infection is probably influenced, not only by immune responses to the virus, but also by the probability that spread of virus from the epithelium to underlying tissues is impeded by noncellular or soluble forms of heparan sulfate or heparin.

\section{Acknowledgments}

Research done by the authors was supported by grants from the NIH (R37 AI-36293, U19 AI-31494, F32 AI-09951). We thank all our colleagues and collaborators in this work.

1. Spear, P.G. 1993. Entry of alphaherpesviruses into cells. Seminars in Virology. 4:167-180.

2. Herold, B.C., WuDunn, D., Soltys, N., and Spear, P.G. 1991. Glycoprotein $C$ of herpes simplex virus type 1 plays a principal role in the adsorption of virus to cells and in infectivity. J. Virol. 65:1090-1098.

3. Herold, B.C., Visalli, R.J., Susmarski, N., Brandt, C.R., and Spear, P.G. 1994. Glycoprotein C-independent binding of herpes simplex virus to cells requires cell surface heparan sulphate and glycoprotein B. J. Gen. Virol. 75:1211-1222.

4. WuDunn, D., and Spear, P.G. 1989. Initial interaction of herpes simplex virus with cells is binding to heparan sulfate. J. Virol. 63:52-58.

5. Shieh, M.-T., WuDunn, D., Montgomery, R.I., Esko, J.D., and Spear, P.G.
1992. Cell surface receptors for herpes simplex virus are heparan sulfate proteoglycans. J. Cell Biol. 116:1273-1281.

6. Gruenheid, S., Gatzke, L., Meadows, H., and Tufaro, F. 1993. Herpes simplex virus infection and propagation in a mouse $\mathrm{L}$ cell mutant lacking heparan sulfate proteoglycans. J. Virol. 67:93-100.

7. Banfield, B.W., Leduc, Y., Esford, L., Schubert, K., and Tufaro, F. 1995. Sequential isolation of proteoglycan synthesis mutants by using herpes simplex virus as a selective agent: evidence for a proteoglycan-independent virus entry pathway. J. Virol. 69:3290-3298.

8. Wei, G., et al. 2000. Location of the glucuronosyltransferase domain in the heparan sulfate copolymerase EXT1 by analysis of Chinese hamster ovary cell mutants. J. Biol. Chem. 275:27733-27740.

9. McCormick, C., et al. 1998. The putative tumour suppressor EXT1 alters the expression of cell-surface heparan sulfate. Nat. Genet. 19:158-161.

10. Nahmias, A.J., and Kibrick, S. 1964. Inhibitory effect of heparin on herpes simplex virus. J. Bacteriol. 87:1060-1066.

11. Tal-Singer, R., et al. 1995. Interaction of herpes simplex virus glycoprotein gC with mammalian cell surface molecules. J. Virol. 69:4471-4483.

12. Williams, R.K., and Straus, S.E. 1997. Specificity and affinity of binding of herpes simplex virus type 2 glycoprotein B to glycosaminoglycans. $J$. Virol. 71:1375-1380.

13. Trybala, E., Liljeqvist, J.-A., Svennerholm, B., and Bergstrom, T. 2000. Herpes simplex virus types 1 and 2 differ in their interaction with heparan sulfate. J. Virol. 74:9106-9114.

14. Svennerholm, B., Jeansson, S., Vahlne, A., and Lycke, E. 1991. Involvement of glycoprotein $\mathrm{C}(\mathrm{gC})$ in adsorption of herpes simplex virus type 1 (HSV-1) to the cell. Arch. Virol. 120:273-279.

15. Gerber, S.I., Belval, B.J., and Herold, B.C. 1995. Differences in the role of glycoprotein C of HSV-1 and HSV-2 in viral binding may contribute to serotype differences in cell tropism. Virology. 214:29-39.

16. Cohen, J.I., and Seidel, K.E. 1994. Absence of varicella-zoster virus (VZV) glycoprotein $\mathrm{V}$ does not alter growth of VZV in vitro or sensitivity to heparin. J. Gen. Virol. 75:3087-3093.

17. Jacquet, A., et al. 1998. The varicella zoster glycoprotein B (gB) plays a role in virus binding to cell surface heparan sulfate proteoglycans. Virus Res. 53:197-207.

18. Compton, T., Nowlin, D.M., and Cooper, N.R. 1993. Initiation of human cytomegalovirus infection requires initial interaction with cell surface heparan sulfate. Virology. 193:834-841.

19. Neyts, J., et al. 1992. Sulfated polymers inhibit the interaction of human cytomegalovirus with cell surface heparan sulfate. Virology. 189:48-58.

20. Kari, B., and Gehrz, R. 1992. A human cytomegalovirus glycoprotein complex designated gC-II is a major heparin-binding component of the envelope. J. Virol. 66:1761-1764.

21. Kari, B., Li, W., Cooper, J., Goertz, R., and Radeke, B. 1994. The human cytomegalovirus UL100 gene encodes the gC-II glycoproteins recognized by group 2 monoclonal antibodies. J. Gen. Virol. 75:3081-3086.

22. Secchiero, P., et al. 1997. Role of the extracellular domain of human herpesvirus 7 glycoprotein $B$ in virus binding to cell surface heparan sulfate proteoglycans. J. Virol. 71:4571-4580.

23. Skrincosky, D., et al. 2000. Identification and analysis of a novel heparinbinding glycoprotein encoded by human herpesvirus 7. J. Virol. 74:4530-4540.

24. Conti, C., et al. 2000. Early interactions of human herpesvirus 6 with lymphoid cells: role of membrane protein components and glycosaminoglycans in virus binding. J. Med. Virol. 62:487-497.

25. Speck, P., Haan, K.M., and Longnecker, R. 2000. Epstein-Barr virus entry into cells. Virology. 277:1-5.

26. Akula, S.M., Wang, F.Z., Vieira, J., and Chandran, B. 2001. Human herpesvirus 8 interaction with target cells involves heparan sulfate. Virology. 282:245-255.

27. Laquerre, S., et al. 1998. Heparan sulfate proteoglycan binding by herpes simplex virus type 1 glycoproteins $B$ and $C$, which differ in their contributions to virus attachment, penetration, and cell-to-cell spread. $J$. Virol. 72:6119-6130.

28. Herold, B.C., Gerber, S.I., Belval, B.J., Siston, A.M., and Shulman, N. 1996. Differences in the susceptibility of herpes simplex virus types 1 and 2 to modified heparin compounds suggest serotype differences in viral entry. J. Virol. 70:3461-3469.

29. Feyzi, E., Trybala, E., Bergstrom, T., Lindahl, U., and Spillmann, D. 1997. Structural requirement of heparan sulfate for interaction with herpes simplex virus type 1 virions and isolated glycoprotein C. J. Biol. Chem. 272:24850-24857.

30. Trybala, E., et al. 1993. Herpes simplex virus type 1-induced hemagglutination: glycoprotein $\mathrm{C}$ mediates virus binding to erythrocyte surface heparan sulfate. J. Virol. 67:1278-1285.

31. Trybala, E., et al. 1994. Localization of a functional site on herpes simplex virus type 1 glycoprotein $\mathrm{C}$ involved in binding to cell surface heparan sulphate. J. Gen. Virol. 75:743-752.

32. Boyle, K.A., and Compton, T. 1998. Receptor-binding properties of a sol- 
uble form of human cytomegalovirus glycoprotein B. J. Virol. 72:1826-1833.

33. Boyle, K.A., Pietropaolo, R.L., and Compton, T. 1999. Engagement of the cellular receptor for glycoprotein B of human cytomegalovirus activates the interferon-responsive pathway. Mol. Cell. Biol. 19:3607-3613.

34. Zhu, H., Cong, J., and Shenk, T. 1997. Use of differential display analysis to assess the effect of human cytomegalovirus infection on the accumulation of cellular RNAs: induction of interferon-responsive RNAs. Proc. Natl. Acad. Sci. USA. 94:13985-13990.

35. Spear, P.G., Eisenberg, R.J., and Cohen, G.H. 2000. Three classes of cell surface receptors for alphaherpesvirus entry. Virology. 275:1-8.

36. Montgomery, R.I., Warner, M.S., Lum, B.J., and Spear, P.G. 1996. Herpes simplex virus-1 entry into cells mediated by a novel member of the TNF/NGF receptor family. Cell. 87:427-436.

37. Geraghty, R.J., Krummenacher, C., Cohen, G.H., Eisenberg, R.J., and Spear, P.G. 1998. Entry of alphaherpesviruses mediated by poliovirus receptorrelated protein 1 and poliovirus receptor. Science. 280:1618-1620.

38. Warner, M.S., et al. 1998. A cell surface protein with herpesvirus entry activity (HveB) confers susceptibility to infection by mutants of herpes simplex virus type 1 , herpes simplex virus type 2 and pseudorabies virus. Virology. 246:179-189.

39. Shukla, D., et al. 1999. A novel role for 3-O-sulfated heparan sulfate in herpes simplex virus 1 entry. Cell. 99:13-22.

40. Lindahl, U., Kusche-Gullberg, M., and Kjellén, L. 1998. Regulated diversity of heparan sulfate. J. Biol. Chem. 273:24979-24982.

41. Shworak, N.W., et al. 1997. Molecular cloning and expression of mouse and human cDNAs encoding heparan sulfate D-glucosaminyl 3-O-sulfotranserase. J. Biol. Chem. 272:28008-28019.

42. Shworak, N.W., et al. 1999. Multiple isoforms of heparan sulfate D-glucosaminyl 3-O-sulfotransferase. J. Biol. Chem. 274:5170-5184.
43. Liu, J., et al. 1999. Expression of heparan sulfate D-glucosaminyl 3-Osulfotransferase isoforms reveals novel substrate specificities. J. Biol. Chem. 274:5185-5192.

44. Willis, S.H., et al. 1998. Examination of the kinetics of herpes simplex virus glycoprotein $\mathrm{D}$ binding to the herpesvirus entry mediator, using surface plasmon resonance. J. Virol. 72:5937-5947.

45. Krummenacher, C., et al. 1999. The first immunoglobulin-like domain of HveC is sufficient to bind herpes simplex virus gD with full affinity, while the third domain is involved in oligomerization of HveC. J. Virol. 73:8127-8137.

46. Liu, J., et al. 1999. Heparan sulfate D-glucosaminyl 3-O-sulfotransferase $3 \mathrm{~A}$ sulfates $\mathrm{N}$-unsubstituted glucosamine residues. J. Biol. Chem. 274:38155-38162.

47. Mocarski, E.S., Jr. 1996. Cytomegaloviruses and their replication. In Fields virology. Volume 2. B.N. Fields, D.M. Knipe, and P.M. Howley, editors. Lippincott-Raven Publishers. Philadelphia, Pennsylvania, USA. 2447-2492.

48. Santoro, F., et al. 1999. CD46 is a cellular receptor for human herpesvirus 6. Cell. 99:817-827.

49. Lusso, P., et al. 1994. CD4 is a critical component of the receptor of human herpesvirus 7: interference with human immunodeficiency virus. Proc. Natl. Acad. Sci. USA. 91:3872-3876.

50. McClain, D.S., and Fuller, A.O. 1994. Cell-specific kinetics and efficiency of herpes simplex virus type 1 entry are determined by two distinct phases of attachment. Virology. 198:690-702.

51. Yura, Y., et al. 1992. Heparan sulfate as a mediator of herpes simplex virus binding to basement membrane. J. Invest. Dermatol. 98:494-498.

52. Huard, J., et al. 1996. The basal lamina is a physical barrier to herpes simplex virus-mediated gene delivery to mature muscle fibers. J. Virol. 70:8117-8123. 